\title{
Mechanical and thermal properties of sugar palm fiber reinforced thermoplastic polyurethane composites: effect of silane treatment and fiber loading
}

\begin{abstract}
ABSTRCT
The aim of the present study was to develop sugar palm fiber (SPF) reinforced thermoplastic polyurethane (TPU) composites and to investigate the effects of fiber surface modification by $2 \%$ silane treatment and fiber loading $(0,10,20,30,40$ and $50 \mathrm{wt} \%)$ on the mechanical and thermal properties of the obtained composites. Surface treatment was employed to improve the fibermatrix interface, which was expected to boost the mechanical strength of the composites, in terms of tensile, flexural and impact properties. Thermal properties were also investigated by thermal gravimetric analysis (TGA) and dynamic mechanical analysis (DMA) to assess the thermal stability of the developed composites. Furthermore, scanning electron microscopy (SEM) was used to study the tensile fracture samples of composites with a view towards evaluating the effects of fiber surface treatments on the fiber/matrix interfacial bonding. The findings of this study reveal that the

silane treatment has determined good bonding and linkage of the cellulose fiber to the TPU matrix, hence contributing to enhanced mechanical and thermal properties of the composites. The composite formulation with $40 \mathrm{wt} \%$ sugar palm fiber loading showed optimum values such as 17.22 MPa for tensile, $13.96 \mathrm{MPa}$ for flexural, and $15.47 \mathrm{~kJ} / \mathrm{m} 2$ for impact strength.

Moreover, the formulations with higher fiber content exhibited satisfactory values of storage modulus and thermal degradation, while their good interfacial adhesion was evidenced by SEM images.
\end{abstract}

Keyword: Sugar palm fibers; Silane treatment; Thermoplastic polyurethane; Sugar palm fiberreinforced composites; Mechanical properties; Thermo-mechanical properties. 